\title{
Using Visco-Plastic Lubrication for Suspension Transportation
}

\author{
Saeed Garmeh, Ali Dolatabadi and Ida Karimfazli \\ Department of Mechanical, Industrial and Aerospace Engineering \\ Concordia University \\ Montreal, Canada
}

\begin{abstract}
This study focuses on a common problem in suspension plasma spraying called clogging. Different mechanisms causing that issue are described and a technique called visco-plastic lubrication is introduced as a solution to this problem. Numerical modelling has been done using mentioned technique and effect of viscosity ratio of the fluids on the flow are investigated.
\end{abstract}

Keywords; clogging; suspension plasma spraying; visco-plastic lubrication; numerical modelling

\section{INTRODUCTION AND BACKGROUND}

Coating plays an important role in surface engineering industries. The aim of coating is to enhance physical and mechanical properties of the material surface [1]. Among different spraying processes, suspension plasma spraying (SPS) is common and popular. In SPS, suspension which carries the material particles that we desire to coat them on top of a substrate, is injected into a plasma torch. These particles melt and stick to the substrate and then they are solidified and adhered to the surface.

Typical suspensions used in SPS have concentration of about $20 \%$ wt. which means only $20 \%$ of the mass of the suspension is composed of particles. This relatively low concentration consumes a lot of energy and time in spraying. One way to reduce the spraying time is to increase the concentration of the particles. For instance, if we use $50 \%$ wt. suspension instead of $20 \%$ wt., we save time and energy with a factor of 2.5 which is significant. However, the chance of agglomeration and clogging that is a common issue in SPS is also raised.

Different ways of clogging occurrence are investigated and a technique called visco-plastic lubrication is introduced and modelled numerically as a solution to the clogging issue. In suspensions, when the concentration of particles is increased, the interaction between particles and the tube wall grows. There are two general ways that cause clogging. (a)

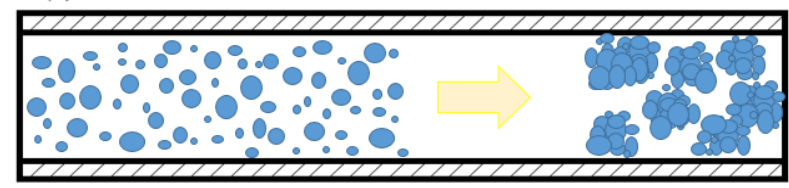

(b)

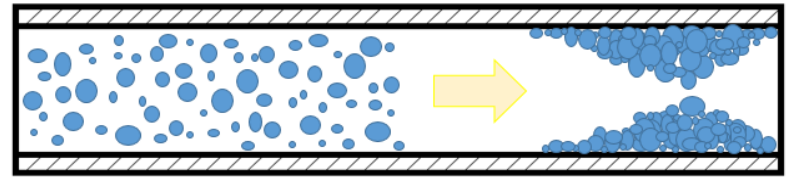

Fig. 1 Schematic of (a) Particle-Particle interactions and (b) Particle-Wall interactions for high concentration suspension inside the tube

Firstly, when the solid particles in the suspension interact with each other, they may adhere together and make bigger agglomerates. These agglomerated parts can be trapped in the carrying tube or injector and cause clogging. A visual representation of this phenomena is shown in Fig. 1(a).

Secondly, when these particles interact with the tube wall and injector, they can be attached to the tube and decrease the passing area gradually to a point that suspension couldn't flow throw the tube and clogging would be the result. Fig. 1(b) shows this phenomena schematically.

For clarifying the problem, possibility of clogging due to the particle-particle interaction was focused. Suspensions of Titanium Dioxide (TiO2) with different concentrations of 20, 30, 40 and $50 \%$ wt. were made and analyzed using laser diffraction system (Malvern Spraytec, UK). Measurement tests presented in Fig. 3 and Table 1 show no considerable difference. If the suspension is prepared properly, for concentration up to $50 \%$, there will be no significant change in particle size distribution. The test indicates the main reason behind the clogging is interaction of particles with the tube wall which is shown in Fig 1(b). This conclusion motivates us to implement a technique for preventing contact of suspension with the tube wall. 
(a)

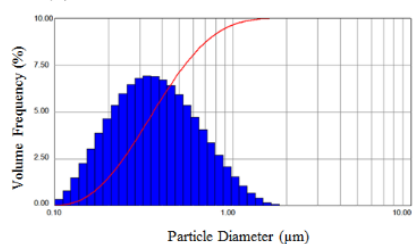

Particle Diameter $(\mu \mathrm{m})$

(c)

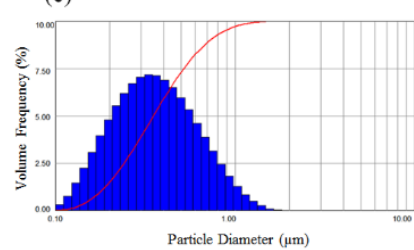

(b)

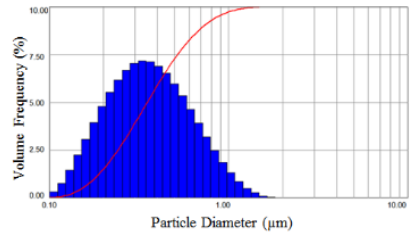

(d)

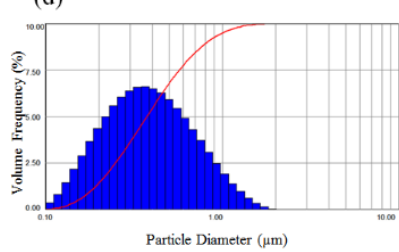

Fig. 2 Particle size distribution for TiO2 suspensions with concentrations of (a) $20 \%$ wt. (b) $30 \%$ wt. (c) $40 \%$ wt. and (d) $50 \%$ wt.

Table 1. Distribution parameters of suspended particles

\begin{tabular}{cccc}
\hline & $\begin{array}{c}\mathbf{D}(\mathbf{1 0}) \\
\boldsymbol{\mu m}\end{array}$ & $\begin{array}{c}\mathbf{D}(\mathbf{5 0}) \\
\boldsymbol{\mu m}\end{array}$ & $\begin{array}{c}\mathbf{D}(\mathbf{9 0}) \\
\boldsymbol{\mu m}\end{array}$ \\
$\mathbf{2 0 \%}$ & 0.1793 & 0.3549 & 0.7391 \\
$\mathbf{3 0 \%}$ & 0.1784 & 0.3512 & 0.7266 \\
$\mathbf{4 0 \%}$ & 0.1782 & 0.3503 & 0.7243 \\
$\mathbf{5 0 \%}$ & 0.1798 & 0.3736 & 0.8196 \\
\hline
\end{tabular}

Proposed solution to this problem is visco-plastic lubrication. In this technique, we have a core annular flow where the core fluid is high concentration suspension and it is lubricated with a yield stress fluid. The outstanding character of yield stress fluids is that they are able to flow only if they are submitted to a stress above a critical value called yield stress of the material. Otherwise they deform like solids [2]. Utilizing yield stress fluid prevents the core fluid to get in contact with the tube wall by preserving an unyielded plug around the core fluid [3]. Fig. 3 shows a schematic of visco-plastic lubrication.

(a)

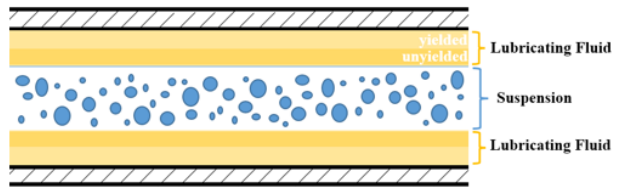

(b)

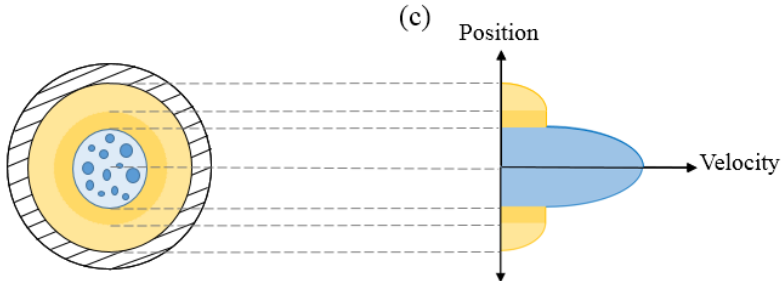

Fig. 3 Schematic of visco-plastic lubrication of suspension in (a) side view, (b) front view and (c) velocity profile of the flow
In this research, $0.3 \%$ Carbomer gel used as lubricating fluid which is a yield stress fluid. Result of the rheology test on the gel and the fitted curves are shown in Fig. 4. The curves generated using MATLAB Curve Fitting tool and the equations of the curves are as follows:

Herschel-Bulkley Model:

$$
\tau=44.18+13.84 \times \dot{\gamma}^{0.2986}
$$

Bingham Model:

$$
\tau=55.12+0.80 \times \dot{\gamma}
$$

Where $\tau$ is the stress and $\dot{\gamma}$ is strain rate. According to (1) and (2), the yield stresses predicted by Herschel-Bulkley and Bingham models are $44.18 \mathrm{~Pa}$ and $55.12 \mathrm{~Pa}$, respectively. As it can be understood from Fig. 4, the Herschel-Bulkley model can predict the behavior of the yield stress fluid more accurately. For calculation of effective viscosity, slope of predicted curve by Bingham model is used when the flow begins [4]. Therefore, $0.80 \mathrm{~Pa}$.s considered as the effective viscosity of the $0.3 \%$ Carbomer Gel.

\section{MECTHODOLOGY:}

\section{A. Numerical model}

All simulations were performed by ANSYS Fluent package. For modelling the multiphase flow, the mixture model was used. This model can be used to model multiphase flows where the phases move at different velocities. The mixture model can model $\mathrm{n}$ phases by solving the momentum, continuity, volume fraction equations for the secondary phases, and algebraic expressions for the relative velocities [5]. The governing equations are as follow:

\section{1) Continuity Equation}

$$
\frac{\partial\left(\rho_{m}\right)}{\partial t}+\nabla \cdot\left(\rho_{m} \vec{v}_{m}\right)=0
$$

Where $\rho_{m}$ is the mixture density according to (4) and $\vec{v}_{\mathrm{m}}$ is the mass-averaged velocity according to (5):

$$
\begin{array}{r}
\rho_{m}=\sum_{k=1}^{n} \alpha_{k} \rho_{k} \\
\vec{v}_{m}=\frac{\sum_{k=1}^{n} \alpha_{k} \rho_{k} \vec{v}_{k}}{\rho_{m}}
\end{array}
$$

Where $\mathrm{n}$ is number of phases. Current numerical study is for a flow of 2 phases. $\alpha_{k}, \rho_{k}$ and $\vec{v}_{k}$ are volume fraction, density and velocity of phase $\mathrm{k}$, respectively. $\mathrm{k}=1$ is representing the lubricating fluid and $\mathrm{k}=2$ is the core fluid.

2) Momentum Equation

$$
\begin{gathered}
\frac{\partial}{\partial t}\left(\rho_{m} \vec{v}_{m}\right)+\nabla \cdot\left(\rho_{m} \vec{v}_{m} \vec{v}_{m}\right)=-\nabla P+\nabla \cdot\left[\mu_{m}\left(\nabla \vec{v}_{m}+\overrightarrow{v_{m}^{T}}\right)\right] \\
+\rho_{m} \vec{g}+\vec{F}+\nabla \cdot\left(\sum_{k=1}^{n} \alpha_{k} \rho_{k} \vec{v}_{d r, k} \vec{v}_{d r, k}\right)
\end{gathered}
$$




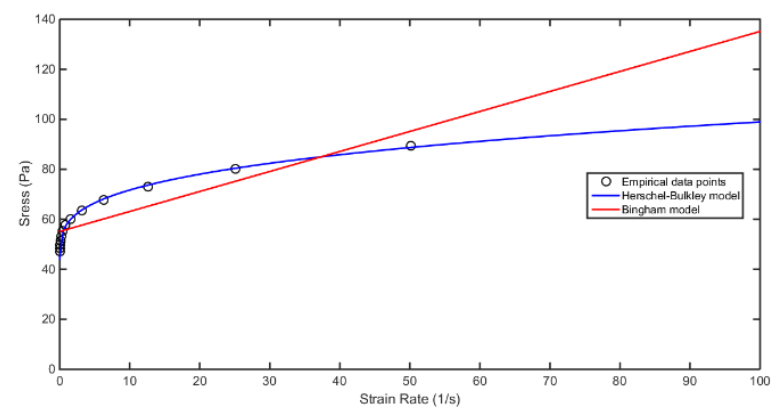

Fig. 4 Flow curve of $0.3 \%$ Carbomer Gel and fitted curves of Bingham and Herschel-Bulkley models

Where $\vec{F}$ is the body force, and $\mu_{m}$ is the viscosity of the mixture:

$$
\mu_{m}=\sum_{k=1}^{n} \alpha_{k} \mu_{k}
$$

And $\vec{v}_{d r, k}$ is the drift velocity for secondary phase $k$ :

$$
\vec{v}_{d r, k}=\vec{v}_{k}-\vec{v}_{m}
$$

\section{3) Volume Fraction Equation for the Secondary Phase}

From the continuity equation for secondary phase $(k=2)$, the volume fraction equation for secondary phase 2 can be obtained:

$$
\begin{gathered}
\frac{\partial}{\partial t}\left(\alpha_{2} \rho_{2}\right)+\nabla \cdot\left(\alpha_{2} \rho_{2} \vec{v}_{m}\right) \\
=-\nabla \cdot\left(\alpha_{2} \rho_{2} \vec{v}_{d r, 2}\right)+\left(\dot{m}_{12}-\dot{m}_{21}\right)
\end{gathered}
$$

Where $\dot{m}_{12}$ is the mass transfer from phase 1 to phase 2 and $\dot{m}_{21}$ is the mass transfer from phase 2 to phase 1 .

\section{B. Dimensional analysis}

According to The Buckingham Pi theorem, groups of dimensionless numbers are presented in Table 2. This study concentrates on investigating the effect of viscosity ratio of core fluid to lubricating fluid on the flow.

Core fluid and lubricating fluid have viscosities of $\mu_{\text {core }}$ and $\mu_{\text {lub }}$, densities of $\rho_{\text {core }}$ and $\rho_{\text {lub }}$ and average velocities of $V_{\text {core }}$ and $V_{\text {lub }}$, respectively. $L$ and $D$ are the length and diameter of the tube and $d_{i}$ is the interface diameter of developed flow. $\tau_{y}$ is the yield stress for lubricating fluid and $\Delta P$ is the pressure drop alongside the tube.

Table 2. Dimensionless groups

\begin{tabular}{|c|c|c|c|}
\hline$\Pi_{1}=\frac{\rho_{\text {core }} V_{\text {core }} d_{i}}{\mu_{\text {core }}}$ & $\Pi_{2}=\frac{\tau_{y} D}{V_{\text {lub }} \mu_{\text {lub }}}$ & $\Pi_{3}=\frac{\Delta P}{\rho_{\text {core }} V_{\text {core }}^{2}}$ & $\Pi_{4}=\frac{\mu_{\text {core }}}{\mu_{\text {lub }}}$ \\
\hline$\Pi_{5}=\frac{V_{\text {core }}}{V_{\text {lub }}}$ & $\Pi_{6}=\frac{\rho_{\text {core }}}{\rho_{\text {lub }}}$ & $\Pi_{7}=\frac{L}{D}$ & $\Pi_{8}=\frac{d_{i}}{D}$ \\
\hline
\end{tabular}

According to table $2, \Pi_{1}$ is the Reynolds number based on the core fluid properties. $\Pi_{2}$ is the Bingham number for lubricating fluid which is the ratio of yield stress to a typical viscous stress of the flow [4]. $\Pi_{3}$ is dimensionless pressure drop based on the core fluid properties, $\Pi_{4}, \Pi_{5}$ and $\Pi_{6}$ are the ratios of core fluid to lubricating fluid properties. $\Pi_{7}$ and $\Pi_{8}$ are groups of the normalized length and interface diameter.

\section{Geometry and computational domain}

The domain inside the tube that is modeled is a cylinder with diameter of $D$ and length of $L$. The grid is made up of quadrilateral cells. ICEM CFD 14.0 was used for generating the mesh. Number of cells used in this study is 211,167 and a grid dependency study has been done to ensure the results are independent from the mesh.

\section{Boundary Conditions:}

This study aims to reveal the relation of viscosity ratio to the flow parameters. Both fluids are injected co-axially to the domain. The velocity inlet boundary condition is assigned for inlets of core and lubricating fluids. Core fluid is injected from the center of cylinder. Pressure outlet used as the boundary condition at the exit of the flow. The wall boundary condition with no-slip is assigned to the tube wall.

In the modelling, the flow rate of each fluid kept at a constant value which results a stable core annular flow. Then, different values were set to the viscosity of the core fluid. By keeping the lubricating fluid parameters constant in all the simulations and just changing the viscosity of the core fluid, different viscosity ratios obtained and simulation repeated for each of those ratios.

\section{E. Simulation results}

Effects of changing the viscosity ratio over dimensionless groups are demonstrated in Fig. 6. Velocity profiles of the flow are also presented in Fig. 7. Note that the velocity and position are normalized by average velocity of lubricating fluid $\left(V_{l u b}\right)$ and diameter of the pipe $(D)$, respectively.

Contours of volume fraction of core fluid in a cut plane alongside the modelled tube for different viscosity ratios are shown in Fig. 8. The tube is long enough to ensure the flow is fully developed.

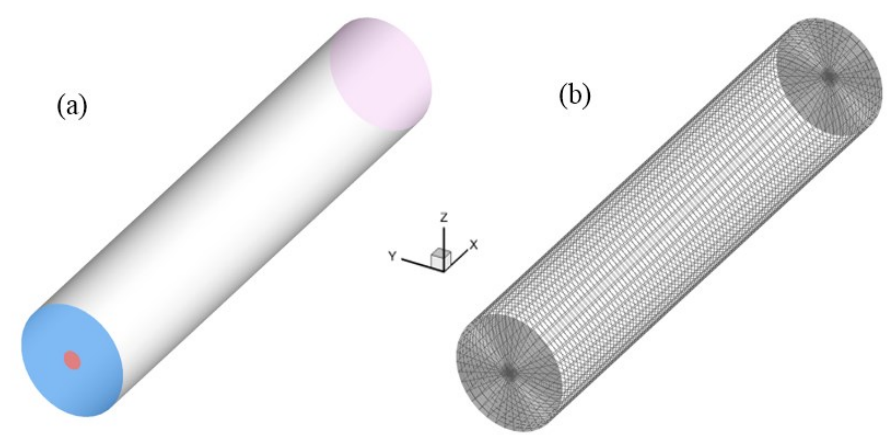

Fig. 5 (a) Geometry and (b) Generated grid 


\section{SUMMARY AND CONCLUSION}

The simulation results showed that using the visco-plastic lubrication technique can prevent suspension to get in contact with the tube wall by forming a solid-like annulus around the core fluid which in fact it can solve the clogging issue. Investigating different viscosity ratios of core fluid to lubricating fluid showed us as the ratio increases, the interface diameter rises which means there is more core fluid in each cross section of the tube. (a)

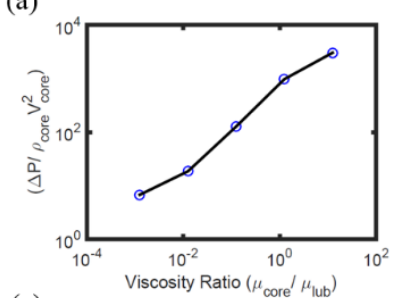

(c)

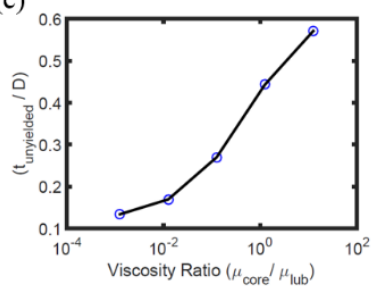

(b)

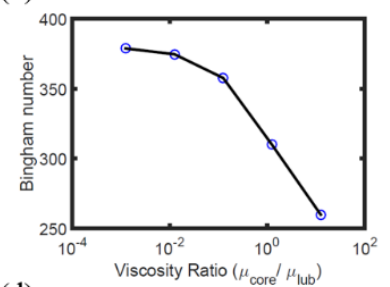

(d)

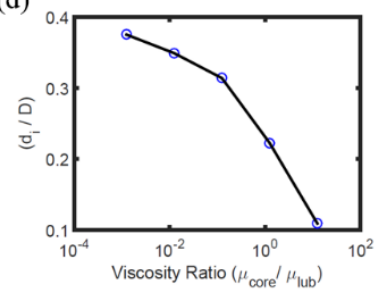

Fig. 6 Effects of changing the viscosity ratio on (a) dimensionless pressure drop (b) Bingham number, (c) normalized thickness of unyielded plug $\left(t_{\text {unyielded }} / D\right)$ around the core fluid and (d) normalized interface diameter $\left(d_{i} / D\right)$ for $L=200$ $\mathrm{mm}, D=38.1 \mathrm{~mm}$ and Reynolds number of $1392,110,6.9,0.41,0.03$ for viscosity ratio of $0.00125,0.0125,0.125,1.25$ and 12.5 , respectively.

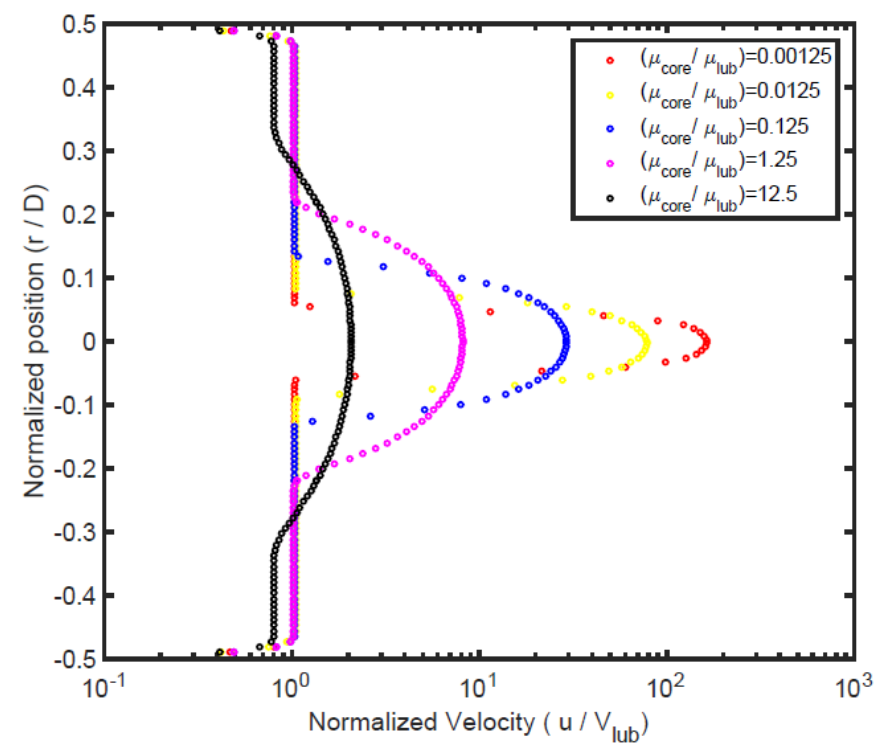

Fig. 7 Velocity profiles for different viscosity ratios

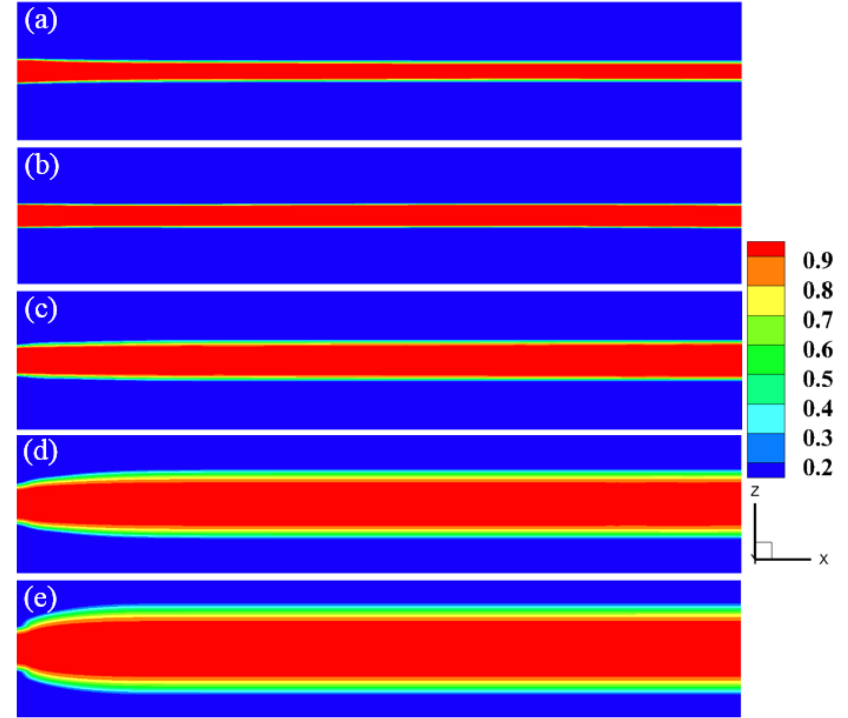

Fig. 8 Contours of volume fraction of core fluid for viscosity ratio of (a) 0.00125 , (b) 0.0125 , (c) 0.125 , (d) 1.25 , (e) 12.5

In all the cases, an unyielded plug was formed around the core suspension. When the viscosity ratio increased, reduction in the thickness of the plug was observed. As long as there is an annulus plug around the core, the interfacial instabilities cannot grow which means the flow is stable. Similar to our expectation, increasing the viscosity of the core fluid resulted in increasing the dimensionless pressure drop $\left(\Pi_{3}\right)$. In addition, increasing the viscosity ratio caused reduction of Bingham number.

Since we are interested in maximizing the flow rate ratio of core fluid to lubricating fluid, the future work of this study will focus on assigning different flow rates for each fluid in order to obtain maximum possible flow rate ratio. In addition, performing experiment in parallel is planned to confirm the simulation results.

\section{ACKNOWLEDGMENT}

The authors acknowledge the financial support from Natural Sciences and Engineering Research Council (NSERC) of Canada.

\section{REFERENCES}

[1] Fauchais, P., A. Joulia, S. Goutier, C. Chazelas, M. Vardelle, A. Vardelle and S. Rossignol (2013). Suspension and solution plasma spraying. Journal of Physics D: Applied Physics. 46: 224015.

[2] Coussot, P. (2014). Yield stress fluid flows: A review of experimental data. Journal of Non-Newtonian Fluid Mechanics. 211: 31-49.

[3] Hormozi, S., D. Martinez and I. Frigaard (2011). Stable core-annular flows of viscoelastic fluids using the visco-plastic lubrication technique. Journal of Non-Newtonian Fluid Mechanics. 166: 1356-1368.

[4] Hormozi, S., K. Wielage-Burchard and I. Frigaard (2011). Entry, start up and stability effects in visco-plastically lubricated pipe flows. Journal of Fluid Mechanics. 673: 432-467.

[5] ANSYS Fluent (2011). Fluent 14 documentation. User's guid 